\title{
混合动力离合器结合过程的动态转矩控制策略*
}

\author{
倪成群 ${ }^{1}$ 张幽䑣 $^{1}$ 赵 强 $^{2}$ BOUKEHILI Adel ${ }^{1}$ \\ (1. 北京理工大学清洁车辆实验室 北京 100081 ; \\ 2. 中国石油管道科技研究中心 廊坊 065000)
}

\begin{abstract}
摘要: 混合动力通过电动机和发动机两种动力源驱动车辆运行, 两种动力源之间的切换对整车的动力性和驾驶性有着重要的 影响。在客车用单轴并联式混合动力系统的基础上, 针对混合动力系统两个动力源响应性差异而造成的整车纵向加速度冲击, 以纯电动模式切换到纯发动机模式和混合驱动模式的过渡过程为研究重点, 提出离合器结合过程的动态转矩控制策略, 即在 离合器结合之前和结合过程中, 采用发动机转速自适应模糊比例积分微分(Proportional-integral-differential, PID)闭环控制跟随 电动机转速; 在离合器结合后, 利用电动机补偿发动机动态转矩, 并且在混合动力系统台架上对提出的动态控制策略进行验 证。试验结果表明, PID 所提出的离合器结合过程的动态转矩控制策略改善了整车纵向冲击度, 提高整车的驾驶性能, 为进 一步的整车试验奠定基础。
\end{abstract}

关键词: 混合动力 动态转矩控制 离合器 控制策略

中图分类号: U467

\section{Dynamic Torque Control Strategy of Engine Clutch in Hybrid Electric Vehicle}

\author{
NI Chengqun ${ }^{1}$ ZHANG Youtong ${ }^{1} \quad$ ZHAO Qiang $^{2} \quad$ BOUKEHILI Adel $^{1}$ \\ (1. Low Emission Vehicle Research Laboratory, Beijing Institute of Technology, Beijing 100081; \\ 2. PetroChina Pipeline R\&D Center, Langfang 065000)
}

\begin{abstract}
Hybrid electric vehicles(HEVs) generate the power required to drive the vehicle via a combination of internal combustion engines and motor, the mode switch between internal combustion engines and motor is essential to vehicle's power and drivability performance. In single-axle parallel hybrid electric vehicle, considering the difference of dynamic characteristics between ICE and motor, there are fluctuation of torque and the longitudinal impact during mode switch, making drivers uncomfortable. Dynamic torque control strategy is designed in order to solve drivability issues caused by propulsion mode's switch during motor-drive mode to engine-drive mode and engine-motor-drive mode. This strategy includes fuzzy adaptive proportional-integral-differential(PID) control for engine speed and dynamic torque compensation control. The control strategy is finally validated by experimental tests based on the test bench of hybrid electric vehicle. The bench test results show that the proposed control strategy can effectively suppress the longitudinal degree of jerk caused by power coupling, which is the fundamental for our deeply research in the future.
\end{abstract}

Key words: Hybrid electric vehicle Dynamic torque control Engine clutch Control strategy

\section{0 前言}

近年来, 由于节能和环保的需求, 新能源汽车 发展迅速, 混合动力兼顾了电动汽车和传统汽车优 点, 因此备受国内外企业以及研究机构的青崃 ${ }^{[1]}$ 。 混合动力系统一般有电动机和发动机等多个动力

*湖北省自然科学基金(2010CDA010)和湖北省中青年创新团队 (T201114)资助项目。20120509 收到初稿, 20121228 收到修改稿
源, 经过离合器或行星齿轮等机构协调组合形成多 种工作模式。不同工作模式之间的切换常带来不同 动力源输出转矩和转速的突变, 从而导致动力输出 的不平稳, 影响整车驾驶性能。如何在保证混合动 力汽车节能减排的同时, 提高模式切换过程中的驾 驶性能, 正受到越来越多的关注 ${ }^{[2]}$ 。

丰田 THS(Toyota hybrid system)系统 ${ }^{[3-4]}$ 采用了 行星齿轮机构的串并联混合动力结构, 发动机的实 际转矩可以由发电动机直接测量, 很好地解决动态 切换过程中的转矩估计和补偿问题。HWANG 等 ${ }^{[5]}$ 
在离合器结合过程中应用发动机和电动机的动态转 矩控制策略, 并在基于 AMESim 软件的混合动力模 型基础上进行仿真研究, 结果表明算法有效提高了 整车的驾驶舒适性。在其他形式的混合动力中, 模 式切换控制研究多采用电动机转矩补偿发动机动态 特性不足的方法。CANOVA 等 ${ }^{[6]}$ 采用基于的模型控 制的方法, 通过电动机快响应来补偿发动机慢响应, 使得发动机起动及运行过程中振动减弱, 提高整车 的驾驶性能。清华大学赵峰等 ${ }^{[7]}$ 针对 ISG 型混合动 力系统, 提出了基于模型匹配 2 自由度控制的动态 协调控制策略, 并进行仿真分析验证了控制策略的 有效性。清华大学童毅等 ${ }^{[8]}$ 及武汉理工大学严运 兵 ${ }^{[9]}$ 提出的 “发动机动态转矩估计+电动机转矩补 偿” 的模式切换动态协调控制策略。但是不论是基 于模型的控制方法还是发动机动态转矩估计, 均采 用复杂的现代控制算法, 不宜在实时控制系统中应 用。同济大学赵治国等 ${ }^{[2]}$ 采用基于混杂系统的无扰 动切换控制算法来控制混合动力模式切换过程, 但 是这种算法对模型要求较高。清华大学戴一凡等 ${ }^{[10]}$ 针对双离合器强混合动力车辆模式切换中系统产生 的冲击, 提出纯电动模式与发动机驱动模式间切换 的转矩协调控制策略, 并进行了台架和实车试验验 证, 达到很好的控制效果。

本文在客车用并联式混合动力系统基础上, 针 对纯电动模式切换到发动机单独驱动模式和混合驱 动模式过程中, 由于两个动力源响应性差异, 所造 成的转矩和转速波动, 提出离合器结合过程中动态 转矩控制策略, 即发动机自适应模糊闭环控制+发 动机动态切换转矩补偿控制策略, 并进行台架试验, 有效地改善整车纵向冲击度, 提高整车的驾驶性能。

\section{1 动力系统}

混合动力系统平台基于天然气-电动机混合动 力城市客车系统, 系统结构如图 1 所示为单轴式混 合动力系统。这种结构的主要特点为天然气发动机、 离合器、电动机和自动机械式变速器(Autornated mechanical transnlission, AMT)采用同轴方式连接。

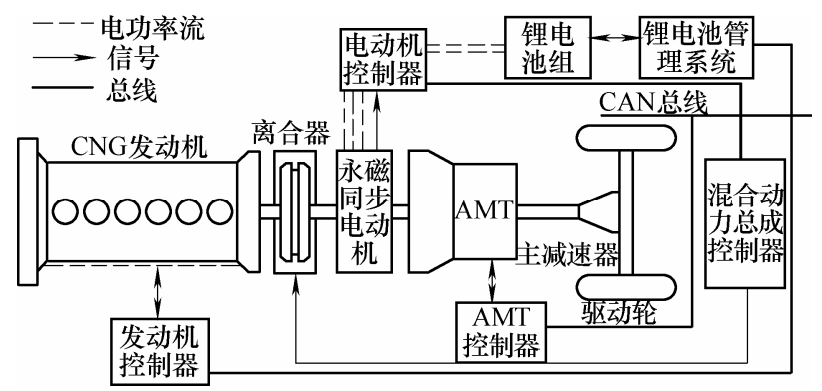

图 1 并联式混合动力系统方案图
并联式混合动力系统主要部件的选型及参数 如表 1 所示。

表 1 混合动力系统主要部件及参数

\begin{tabular}{|c|c|c|c|}
\hline \multirow{2}{*}{ 部件名称 } & \multirow{2}{*}{ 类型 } & \multicolumn{2}{|c|}{ 主要技术参数 } \\
\hline & & 参数 & 数值 \\
\hline \multirow{2}{*}{ 发动机 } & \multirow{2}{*}{ 天然气发动机 } & 功率 $P_{\mathrm{e}} / \mathrm{kW}$ & 172 \\
\hline & & 峰值转矩 $T_{\max } /(\mathrm{N} \cdot \mathrm{m})$ & 678 \\
\hline \multirow{2}{*}{ 电动机 } & \multirow{2}{*}{$\begin{array}{l}\text { 内置式永磁同步 } \\
\text { 电动机 }\end{array}$} & 功率 $P_{\mathrm{e}} / \mathrm{kW}$ & 115 \\
\hline & & 峰值转矩 $T_{\max } /(\mathrm{N} \cdot \mathrm{m})$ & 540 \\
\hline \multirow{2}{*}{ 蓄电池 } & \multirow{2}{*}{ 锂电锂电池组 } & 额定电压 $U / \mathrm{V}$ & 288 \\
\hline & & 容量 $Q /(\mathrm{A} ・ \mathrm{~h})$ & 180 \\
\hline \multirow{6}{*}{ 变速器 } & \multirow{6}{*}{ 綦江 S5-80AMT } & 1 挡速比 $i_{1}$ & 6.90 \\
\hline & & 2 挡速比 $i_{2}$ & 3.83 \\
\hline & & 3 挡速比 $i_{3}$ & 2.32 \\
\hline & & 4 挡速比 $i_{4}$ & 1.49 \\
\hline & & 5 挡速比 $i_{5}$ & 1.00 \\
\hline & & 倒挡速比 $i_{\mathrm{R}}$ & 5.89 \\
\hline
\end{tabular}

混合动力控制系统中, 混合动力总成控制器通 过 CAN 总线对电动机控制器、发动机控制器、AMT 控制器(兼容控制离合器)和电池管理系统进行协调 管理。

\section{2 控制策略}

\section{1 混合动力系统工作模式}

混合动力系统采用基于规则的转矩分配整车 能量管理策略 ${ }^{[11]}$, 即根据发动机和电动机稳态万有 特性图, 以发动机和电动机转矩为直接控制对象, 在不影响驾驶性能的前提下, 采用最佳的转矩分配 规则控制系统运行。

在低速和低负荷时, 离合器分离, 电动机单独 驱动车辆, 这样就可以减少急速工况。发动机工作 时, 可以通过电动机助力或电动机充电来调节发动 机工作区域, 使得发动机工作在高效区。除此之外, 减速或制动时采用主动能量回收, 提高混合动力系 统燃油经济性。

基于系统方案和上述系统功能的分析, 可确定 混合动力系统的工作模式主要有以下几种: 纯电动 模式、发动机单独驱动模式、发动机驱动并发电模 式、混合驱动模式和制动模式等。各个模式下主要 部件的工作模式如表 2 所示。

表 2 混合动力系统工作模式

\begin{tabular}{lccc}
\hline \multicolumn{1}{c}{ 工作模式 } & 发动机状态 & 电动机状态 & 离合器状态 \\
\hline 纯电动 & 不工作 & 电动 & 分离 \\
发动机单独驱动 & 工作 & 不工作 & 结合 \\
发动机驱动并发电 & 工作 & 发电 & 结合 \\
混合驱动 & 工作 & 电动 & 结合 \\
制动能量回收 & 不工作 & 发电 & 分离 \\
\hline
\end{tabular}


从表 2 中可知, 混合动力系统的两个动力源, 经过离合器协调可以组合形成多种工作模式, 不同 状态之间的切换往往需要离合器参与。其中纯电动 模式切换到发动机参与的驱动模式时, 由于天然气 发动机转矩动态响应性较慢 (与同等柴油机相比), 往往在离合器结合过程及结合后发动机转矩恢复 中, 动力输出轴会产生冲击, 并且这种冲击随着整 车负荷增大而增加, 影响整车的驾驶舒适性。针对 此种动力耦合过程中问题, 本文提出了离合器结合 动态转矩协调控制策略。

\section{2 离合器结合动态转矩协调控制策略}

混合动力离合器结合动态转矩控制策略可以 归结如下: 通过协调电动机和发动机输出转矩的方 式来进行, 即在离合器结合之前和结合过程中, 发 动机转速自适应模糊闭环控制跟随电动机驱动车辆 转速, 在离合器结合后由于发动机转矩响应远慢于 电动机转矩响应, 所以采用动态切换转矩补偿控制 策略, 即采用电动机转矩补偿发动机转矩输出的滞 后, 以达到离合器结合动态转矩协调控制的实现。

\subsection{1 发动机转速闭环控制策略}

在离合器结合之前和结合过程中, 起动以后的 发动机转速通过闭环控制跟随电动机驱动车辆的转 速, 虽然天然气发动机电控系统具有转速闭环控制 模式, 但是在中低速、低温和低负荷的情况下, 转 速闭环控制模式下发动机输出实际转速波动较大, 这样就不能满足发动机转速快速跟随的要求。因此 本文的控制策略主要是直接控制发动机转矩达到对 发动机转速闭环控制。由于发动机本身具有非线性、 多工况、时变时滞等特点, 不同的环境状态下发动 机的快速转速闭环控制变得十分困难。传统的闭环 控制多数采用比例积分微分 (Proportional-integraldifferential, PID)控制, 因其具有结构简单、稳定性 好等优点。但是 PID 控制中参数的整定是影响控制 器的关键因素。一般来讲, 可以通过数学模型和试 验调试结合的方法整定出合适的参数。在负荷扰动 等因素的影响下, 过程参数会亦会随工作环境和时 间的变化而变化。这就对 PID 控制器提出在线整定 的要求。

综上考虑，本文采用自适应模糊控制的方法 ${ }^{[12]}$ 在线整定 PID 参数, 这样既能够适应发动机时变性, 又可以继承传统 PID 的优点。发动机自适应模糊 PID 控制结构图如图 2 所示, 以电动机驱动车辆转 速为设定转速与发动机的实际转速误差 $e$ 和一阶差 分 $\Delta e$ 为输入, 首先对他们进行模糊量化, 然后经过 模糊推理规则得出模糊输出值, 对于发动机转速控 制中一般采用 PI 控制即可, 最后反模糊化后得到精
确的 PI 控制器参数。

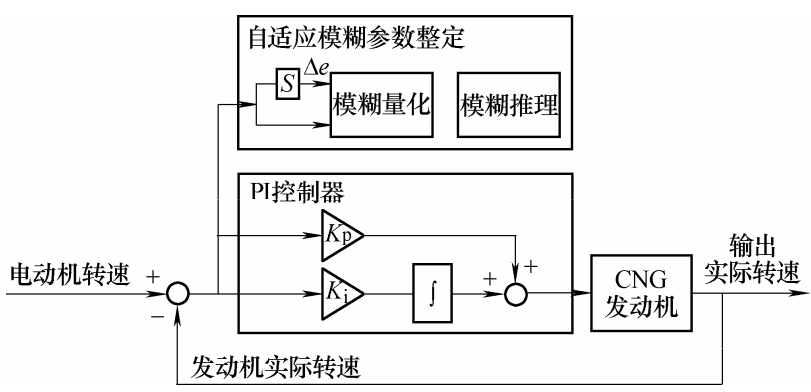

图 2 发动机自适应模糊 PID 控制结构图

假定 PI 控制器中比例增益系数和微分增益系 数分别用 $K_{\mathrm{p}}$ 和 $K_{\mathrm{i}}$ 来表示, 为了简化计算, 基于相 似理论将参数进行归一化处理。 $K_{\mathrm{p}}$ 和 $K_{\mathrm{i}}$ 通过式(1) 和式(2)转化为 $0 \sim 1$ 的参数 $K_{\mathrm{p}}^{\prime}$ 和 $K_{\mathrm{i}}^{\prime}$ 。

$$
\begin{gathered}
K_{\mathrm{p}}^{\prime}=\frac{K_{\mathrm{p}}-K_{\mathrm{p}, \min }}{K_{\mathrm{p}, \text { max }}-K_{\mathrm{p}, \text { min }}} \\
K_{\mathrm{i}}^{\prime}=\frac{K_{\mathrm{i}}-K_{\mathrm{i}, \text { min }}}{K_{\mathrm{i}, \max }-K_{\mathrm{i}, \text { min }}}
\end{gathered}
$$

式中, $K_{\mathrm{p}, \text { max }}, K_{\mathrm{p}, \text { min }}, K_{\mathrm{i}, \text { max }}$ 和 $K_{\mathrm{i}, \text { min }}$ 分别由试验得 到的 $K_{\mathrm{p}}$ 和 $K_{\mathrm{i}}$ 的最大值和最小值。

隶属度函数的确定需要对模糊控制器的输入 和输出变量空间做模糊分割, 重叠的部分意味着模 糊控制规则间模糊的程度, 对控制器性能影响很大, 采用试验调整的方式来确定分割方式, 最终选择比 例增益系数 $K_{\mathrm{p}}^{\prime}$ 为梯形隶属函数, 如图 3 所示, 积 分增益系数 $K_{\mathrm{i}}^{\prime}$ 的隶属函数与之相同。转速误差 $e$ 的隶属函数如图 4 所示, 其采用的模糊集为 $\{\mathrm{NB}$, $\mathrm{NS}, \mathrm{ZO}, \mathrm{PS}, \mathrm{PB}\}$ 的三角形隶属函数, 一阶差分 $\Delta e$ 的隶属函数与之相同。

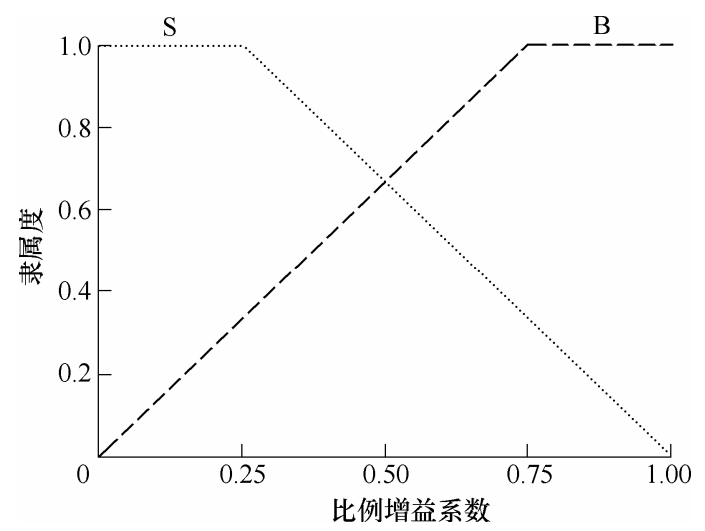

图 3 比例增益系数的隶属函数

模糊规则的确定必须考虑系统响应性能及避 免系统出现超调和振荡。基于以上考虑, 表 3 和表 4 分别为 $K_{\mathrm{p}}^{\prime}$ 和 $K_{\mathrm{i}}^{\prime}$ 的模糊推理规则。经过模糊规则 推理得到模糊控制器输出结果, 不能直接控制被控 对象, 需要进行反模糊化, 采用重心法来进行解模 糊, 输出结果即是 PI 控制器的控制参数。在天然气 
发动机的试验基础上, 得出 $K_{\mathrm{p}}$ 和 $K_{\mathrm{i}}$ 的取值范围, 并且结合模糊 PID 控制, 通过试验验证发动机转速 跟随特性得到明显改善。

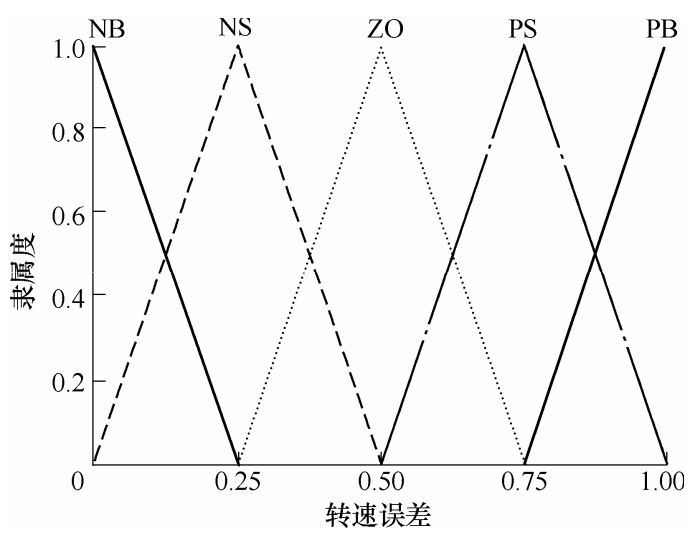

图 4 转速误差隶属函数

表 $3 K_{\mathrm{p}}^{\prime}$ 模糊调整规则

\begin{tabular}{ccccccc}
\hline & & \multicolumn{5}{c}{ 转速误差一阶差分 $\Delta e$} \\
\cline { 3 - 7 } & & $\mathrm{NB}$ & $\mathrm{NS}$ & $\mathrm{ZO}$ & $\mathrm{PS}$ & $\mathrm{PB}$ \\
\hline \multirow{4}{*}{ 转速 } & $\mathrm{NB}$ & $\mathrm{B}$ & $\mathrm{B}$ & $\mathrm{B}$ & $\mathrm{B}$ & $\mathrm{B}$ \\
误差 $e$ & $\mathrm{NS}$ & $\mathrm{S}$ & $\mathrm{B}$ & $\mathrm{B}$ & $\mathrm{B}$ & $\mathrm{S}$ \\
& $\mathrm{PS}$ & $\mathrm{S}$ & $\mathrm{S}$ & $\mathrm{B}$ & $\mathrm{S}$ & $\mathrm{S}$ \\
& $\mathrm{PB}$ & $\mathrm{B}$ & $\mathrm{B}$ & $\mathrm{B}$ & $\mathrm{B}$ & $\mathrm{S}$ \\
& & & & & $\mathrm{B}$ & $\mathrm{B}$ \\
\hline
\end{tabular}

表 $4 K_{i}^{\prime}$ 模糊调整规则

\begin{tabular}{ccccccc}
\hline & \multicolumn{5}{c}{ 转速误差一阶差分 $\Delta e$} \\
\cline { 3 - 7 } & & NB & NS & ZO & PS & PB \\
\hline \multirow{4}{*}{ 转速 } & NB & B & S & S & S & B \\
误差 $e$ & ZO & B & B & S & B & B \\
& PS & B & B & B & B & B \\
& PB & B & S & S & B & B \\
\hline
\end{tabular}

\subsection{2 动态切换转矩补偿控制策略}

在离合器结合之后, 混合动力系统切换到发动 机单独工作模式, 由于发动机的时滞性, 不能很快 响应整车控制器的转矩需求(天然气发动机尤其突 出), 就会带来驱动系统转矩波动, 影响驾驶性能。

由此本文引入动态切换过程转矩补偿控制策 略。如图 5 所示, 控制策略中发动机采用转矩开环 控制, 发动机设定转矩经过驾驶员需求处理后与巡 航控制输出转矩和路面转速限制管理输出转矩进行 比较后得到驾驶员需求的转矩, 经过发动机控制模 式处理计算, 然后与急速管理系统和全负荷限制比 较后输出发动机实际转矩。其中发动机实际输出转 矩均可以通过 CAN 总线直接得到, 但是发动机的 实际输出的转矩和发动机设定转矩存在一定的滞 后。由于电动机采用转矩闭环控制, 并且响应性较 好, 因此考虑用电动机来实时补偿天然气发动机。

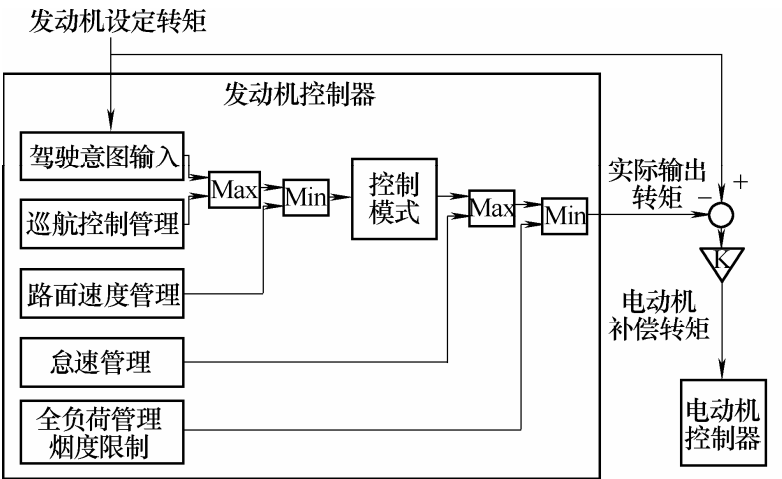

图 5 动态切换转矩补偿控制策略结构图

动态切换转矩补偿控制策略主要从离合器结 合到发动机实际转矩达到发动机设定转矩的区间 内, 采用发动机实际转矩与设定转矩之间的差值由 电动机转矩补偿。如式(3)所示, 电动机转矩 $T_{\mathrm{m}, \text { target }}$ 等于发动机设定转矩 $T_{\mathrm{e}, \mathrm{set}}$ 与发动机实际转矩 $T_{\mathrm{e}}$ 之差。

$$
T_{\mathrm{m}, \mathrm{target}}=T_{\mathrm{e}, \mathrm{set}}-T_{\mathrm{e}}
$$

由于策略中的发动机实际转矩是由发动机控 制器直接输出, 发动机输出实际转矩的估计可能存 在偏差, 这就势必影响整个补偿策略的精确度。因 此在式(3)的基础上，并且考虑切换过程中的实际情 况, 对发动机实际转矩和设定转矩的差值进行修正, 最后得到如式(4)所示

$$
T_{\mathrm{m}, \text { target }}=K\left(T_{\mathrm{e}, \text { set }}-T_{\mathrm{e}}\right)
$$

式中, $K$ 为动态切换补偿切换转矩修正系统。

\section{3 试验研究}

\section{1 台架组成}

为了验证本文的控制策略, 结合上文提出的混 合动力系统构型, 建立单轴混合动力系统的试验台 架, 其中试验台架布置方案如图 6a 所示, 台架实物 如图 $6 \mathrm{~b}$ 所示。台架采用同轴的方式将天然气发动 机、离合器、电动机、 $\mathrm{AMT}$ 及测功机连接起来, 电 池组通过电动机控制器驱动电动机运行。

\section{2 台架试验}

在台架基础上，为了达到对控制策略快速可靠 的验证，控制策略的试验采用先进的快速控制原型 方法, 即利用 dSPACE MicroAutobox 代替整车控制 器对混合动力系统进行控制。如需要进一步应用到 整车控制器中可以通过自动代码生成工具生成 $\mathrm{C}$ 代 码, 再下载到整车控制器中进行控制, 简化开发流 程。针对上文中提出的离合器结合之前和结合过程 中发动机转速闭环控制策略和离合器结合后动态切 


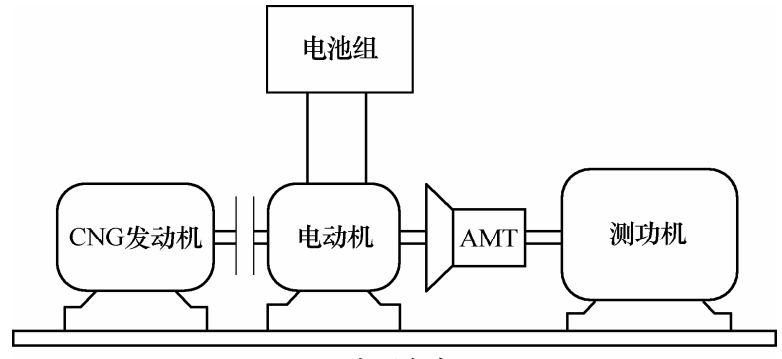

(a) 布置方案

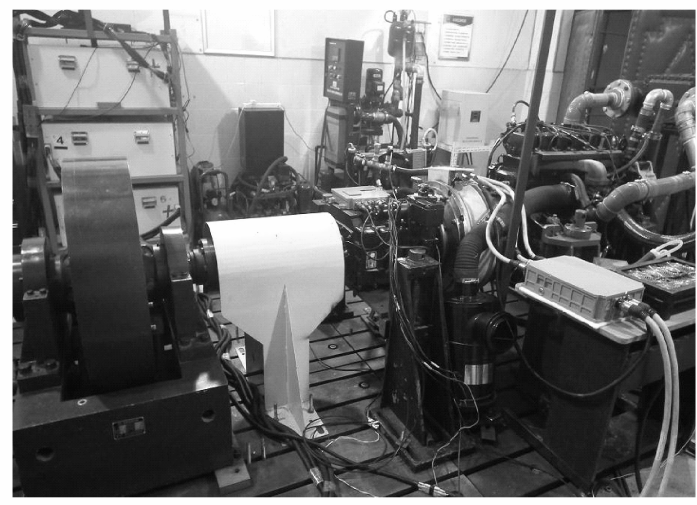

(b) 实物

图 6 试验台架的布置方案及实物图

换转矩补偿控制策略, 本文中设计了四种试验, 分 别为无控制策略试验、发动机转速闭环控制策略试 验、动态切换转矩补偿控制策略试验及两种控制策 略结合试验。

试验工况采用局部工况来对算法进行验证, 主 要考虑纯电动模式切换到发动机单独驱动模式和混 合驱动模式, 制动模式切换到发动机单独驱动模式 和混合驱动模式。其中制动模式切换到发动机单独 驱动模式和混合驱动模式, 在本算法适用的范围内 的切换过程与纯电动模式的切换情况大致相同, 因 此, 本试验中采用纯电动模式切换到发动机单独驱 动模式, 纯电动模式切换到混合驱动模式两种切换 模式进行验证。试验过程中, 选用混合动力客车运 行中比较典型的切换工况, 纯电动模式切换到发动 机单独驱动模式中纯电动工况下恒定电动机转矩为 $100 \mathrm{~N} \cdot \mathrm{m}$, 当电动机转速达到 $1200 \mathrm{r} / \mathrm{min}$ 时开始切 换, 切换后发动机的设定转矩亦为 $100 \mathrm{~N} \cdot \mathrm{m}$ 。纯电 动模式切换到混合驱动模式中纯电动工况下恒定电 动机转矩为 $120 \mathrm{~N} \cdot \mathrm{m}$, 当电动机转速达到 1200 $\mathrm{r} / \mathrm{min}$ 时开始切换, 切换后发动机的设定转矩为 100 $\mathrm{N} \cdot \mathrm{m}$, 电动机转矩为 $20 \mathrm{~N} \cdot \mathrm{m}$ 。变速器选择平路起 步挡位 2 挡。在整个试验过程中测功机负载恒定。

\subsection{1 无控制策略试验}

由纯电动机工作状态切换到发动机单独工作 状态的过程, 在无任何控制策略的情况下, 如图 $7 \mathrm{~b}$ 所示, 切换开始时, 发动机设定的转矩增加, 当发 动机转速与电动机转速相等后, 控制离合器结合, 离合器结合后发动机提供驱动转矩, 并且电动机转
矩降到零。在整个过程中(图 7), 由于发动机转矩响 应的时滞性, 当发动机转速和电动机转速相等后, 发动机转速会继续上升, 使得在离合其结合过程中, 动力输出端转速波动较大。同时在离合器结合后, 由于不能及时响应发动机设定转矩, 也会产生动力 输出端转速波动。整个过程中动力输出轴产生正向 和负向三个数值较大的冲击度, 对驾驶的舒适性有 较大影响。

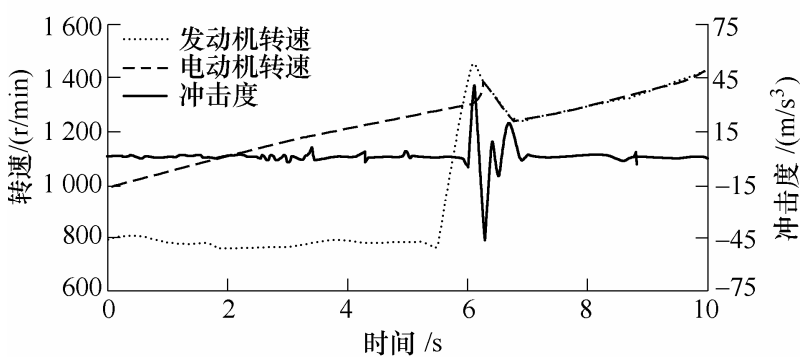

(a)

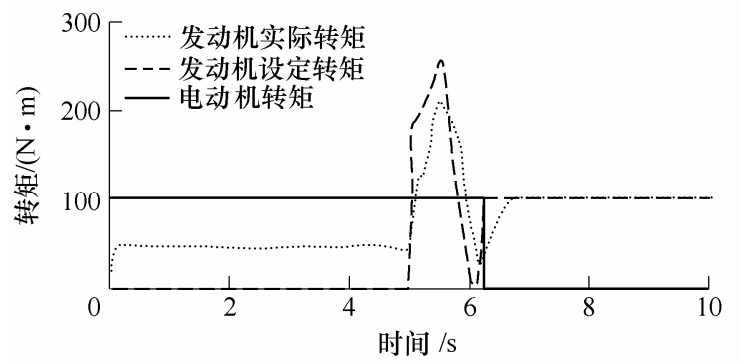

(b)

图 7 无控制策略中切换至纯发动机试验结果

图 8 所示在无任何控制策略的情况下, 由纯电 动模式切换至混合驱动模式的试验结果, 切换过程 中转矩变化如图 $8 \mathrm{~b}$ 所示。图 $8 \mathrm{a}$ 为切换过程中转速 和冲击度变化, 整个切换过程中动力输出轴同样产 生正向和负向三个数值较大冲击度, 影响驾驶性。

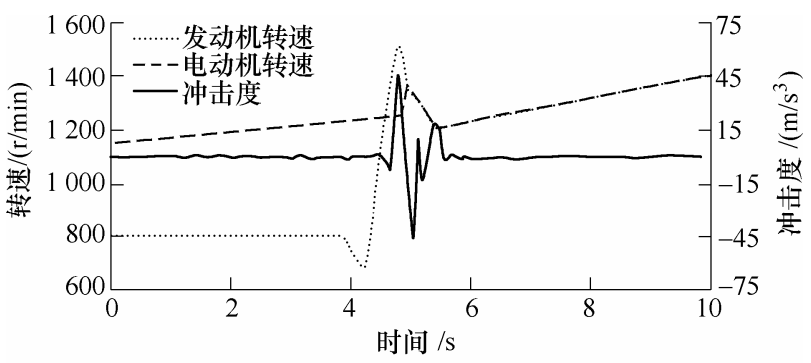

(a)

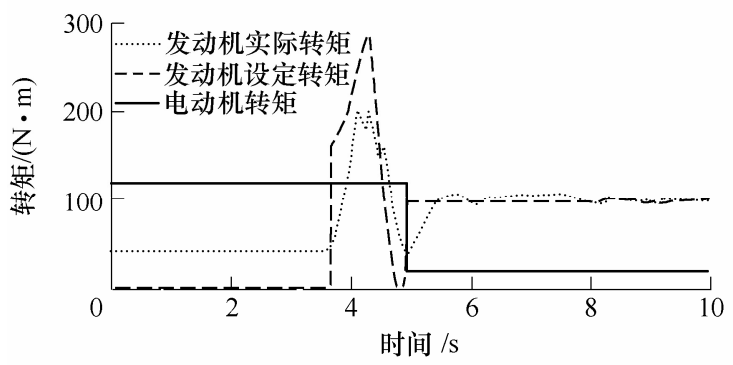

(b)

图 8 无控制策略中切换至混动模式试验结果 


\subsection{2 发动机转速闭环控制策略试验}

在离合器结合之前和结合过程中, 起动以后的 发动机, 通过自适应模糊闭环控制跟随电动机驱动 车辆转速, 纯电动机模式切换到纯发动机驱动模式 的试验结果由图 9 所示, 其中发动机转速、电动机 转速和动力输出轴冲击度如图 $9 \mathrm{a}$ 所示, 与图 7a 相 比可知, 所设计的自适应模糊 PI 控制器, 在离合器 结合过程中, 有效地减少动力输出轴冲击度, 但是 离合器结合后动力输出轴转速波动依然明显。其中 图 $9 \mathrm{~b}$ 所示为发动机设定转矩和实际转矩及电动机 转矩。

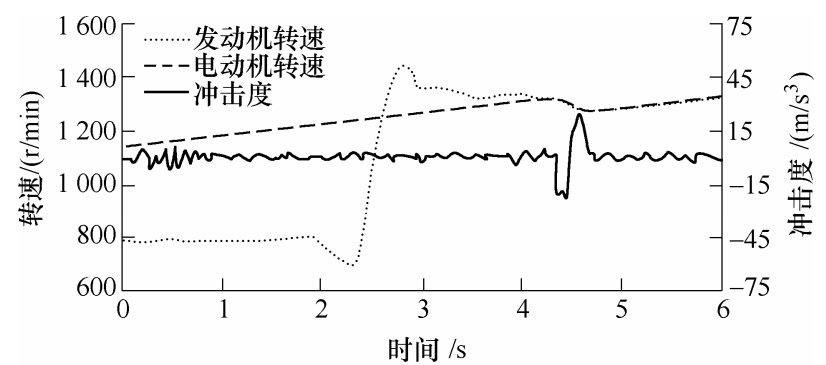

(a)

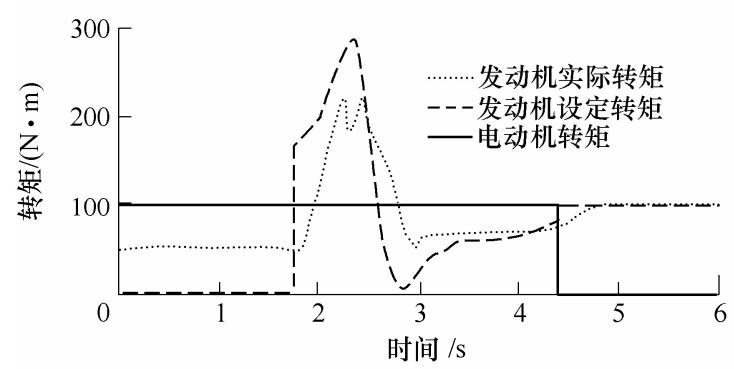

(b)

图 9 发动机转速闭环控制中切换至纯发动机的试验结果

图 10 所示为发动机转速闭环控制策略中, 由 纯电动模式切换至混合驱动模式的试验结果, 与图

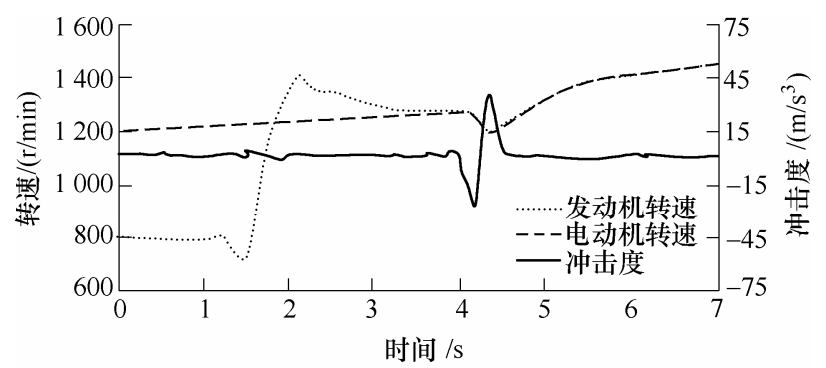

(a)

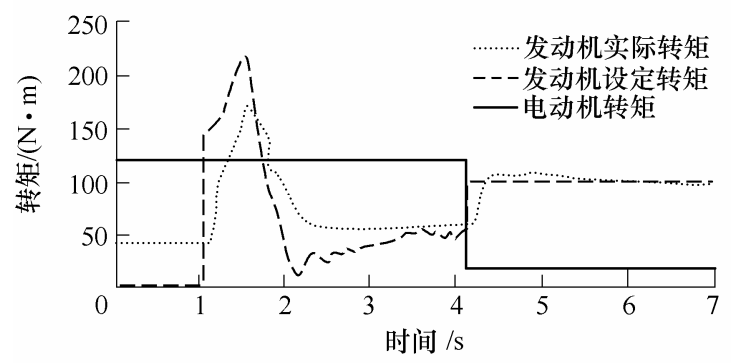

(b)

图 10 发动机转速闭环控制中切换至混动的试验结果
8 相比可知, 所设计的自适应模糊 PI 控制器, 在离 合器结合过程中, 同样减少了动力输出轴冲击度, 但是离合器结合后动力输出轴转速波动依然明显。 影响车辆的驾驶性能。

\subsection{3 动态切换转矩补偿控制策略试验}

在离合器结合后采用发动机动态切换转矩补 偿控制策略, 纯电动机模式切换到纯发动机驱动模 式的试验结果如图 11 所示, 其中发动机转速、电动 机转速和动力输出轴加速度如图 11a 所示, 与图 $7 \mathrm{a}$ 相比, 发动机动态切换转矩补偿控制策略使得离合 器结合前后车辆的纵向冲击度值有所减小, 转速波 动幅度明显有所改善。但是离合器结合过程中发动 机转速和电动机转速存在差异, 带来结合过程中转 速的波动, 同样会使得驾驶性变差。发动机设定转 矩和实际转矩及电动机转矩如图 $9 b$ 所示。离合器结 合后电动机的动态转矩补偿系数设为 1.2 。

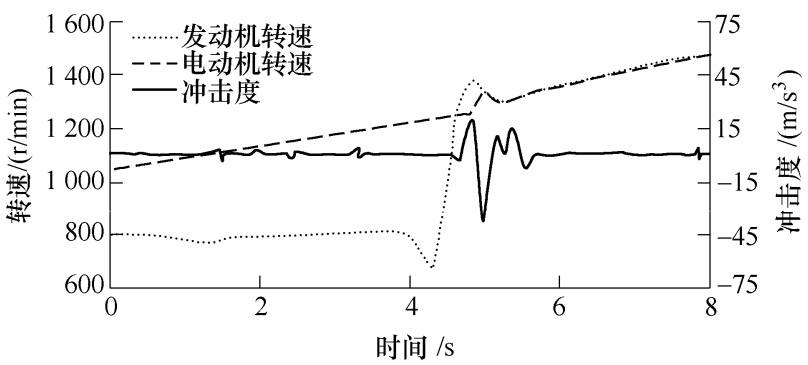

(a)

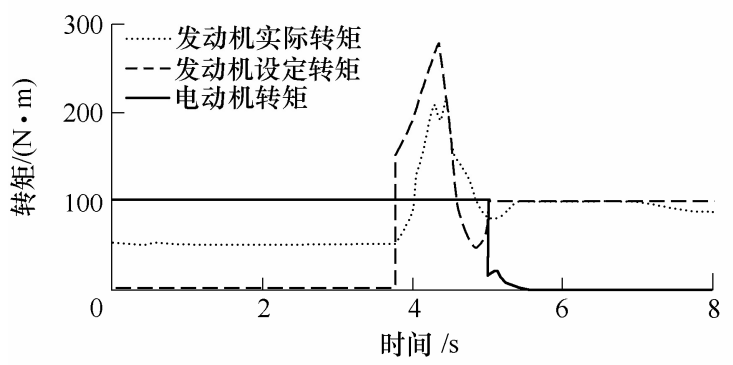

(b)

图 11 动态切换转矩补偿控制中切换至纯发动机试验结果

图 12 所示为发动机动态切换转矩补偿控制策 略中, 由纯电动模式切换至混合驱动模式的试验结 果, 与图 8 相比, 发动机动态切换转矩补偿控制策 略使得离合器结合前后车辆的纵向冲击度值有所减 小，虽然冲击度减少不是很明显，但是动力输出轴 转速波动幅度明显有所改善。冲击度的产生主要是 由于发动机转速和电动机转速存在差异, 在离合器 结合过程中引起动力输出轴转速突变。

\subsection{4 两种控制策略结合试验}

图 13、14 为上文所设计两种控制策略相结合 的离合器结合过程中动态转矩控制策略, 分别在纯 电动模式切换至纯发动机驱动, 纯电动模式切换至 混合驱动模式的试验结果。从图 13、14 可以看出, 


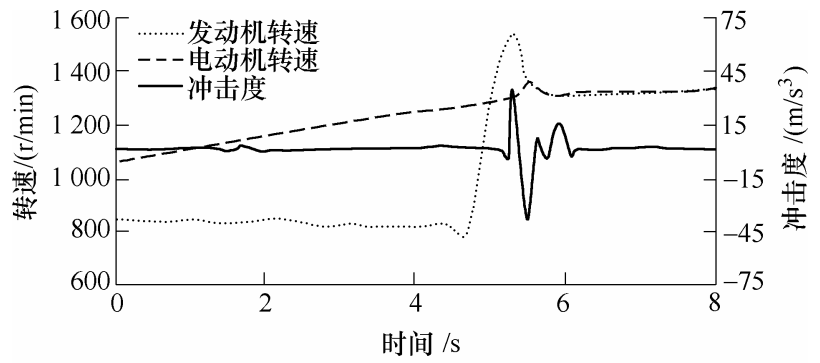

(a)

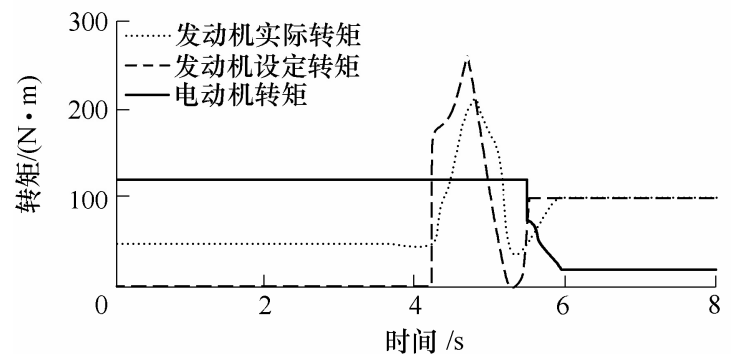

(b)

图 12 动态切换转矩补偿控制中切换至混动的试验结果

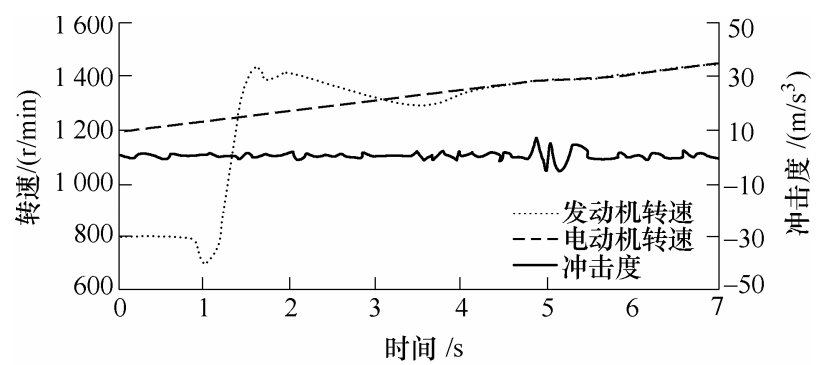

(a)

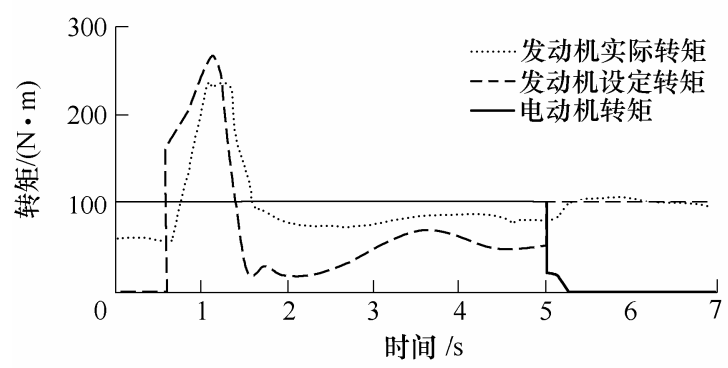

(b)

图 13 两种控制策略结合中切换至纯发动机的试验结果

整个过程中冲击度明显得到改善，但是切换时间有 所增加, 主要是由于天然气发动机调速时间较长, 可以考虑增加天然气发动机的响应性, 或通过离合 器滑磨控制减小冲击, 两种方案均有待进一步研究。

表 5 为四种试验方案所产生的最大冲击度对比 表，其中从纯电动模式切换至纯发动机模式为试验 组 1 , 从纯电动模式切换至混动模式为试验组 2 。一 般来讲, 车辆产生的冲击度上限值为 $10 \mathrm{~m} / \mathrm{s}^{3}$, 超过 此值后乘客感觉不舒服。从表 5 中可以看出, 无论 是从纯电动模式切换到纯发动机模式还是混动模 式, 两种控制结合的离合器结合过程中动态转矩控 制策略基本满足车辆冲击度的要求, 与其他三种试 验方案比较, 在改善车辆冲击度方面作用明显。

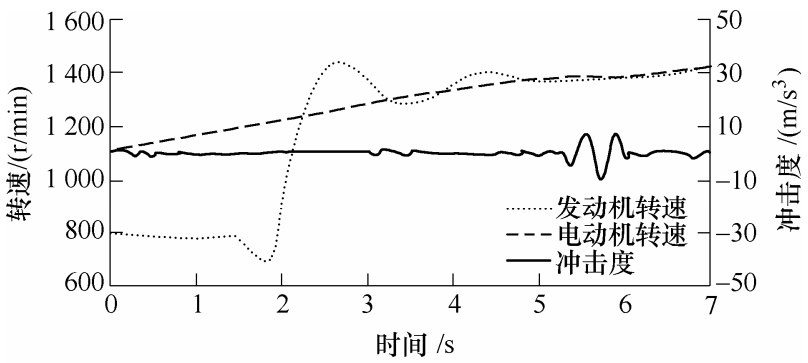

(a)

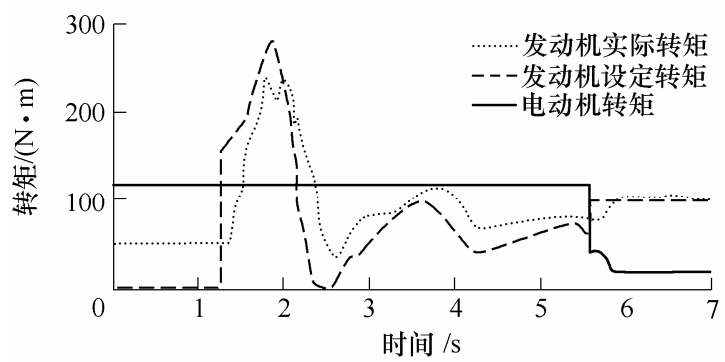

(b)

图 14 两种控制策略结合中切换至混动的试验结果

表 5 各试验方案最大冲击度统计结果

\begin{tabular}{lcc}
\hline \multirow{2}{*}{ 试验项目 } & \multicolumn{2}{c}{ 最大冲击度 $/\left(\mathrm{m} / \mathrm{s}^{3}\right)$} \\
\cline { 2 - 3 } & 试验组 1 & 试验组 2 \\
\hline 无控制策略试验 & 46.5 & 44.7 \\
发动机转速闭环控制策略试验 & 24.0 & 35.4 \\
动态切换转矩补偿控制策略试验 & 37.3 & 39.8 \\
两种控制策略结合试验 & 8.9 & 9.1 \\
\hline
\end{tabular}

\section{4 结论}

本文针对设计的单轴混合动力客车系统, 搭建 混合动力系统台架, 设计了离合器结合过程中动态 转矩控制策略, 基于 dSPACE MicroAutobox 控制平 台进行了控制策略台架性能的试验验证。

(1) 台架试验结果表明, 所设计的离合器结合 过程中动态转矩控制策略有效地抑制了动力耦合过 程中的转速波动, 减小了模式切换造成的整车纵向 冲击度，改善整车的驾驶性能。

(2) 所应用的控制策略在切换过程中使得车辆 冲击度得到改善, 但是切换时间有所增加, 主要是 由于天然气发动机调速时间较长, 下一步应进一步 进行优化研究。

(3) 由于台架系统中的测功机为电涡流测功 机, 不能的模拟路面的负载变化的情况, 本试验只 是在恒定负载下进行的, 因此考虑在整车系统中对 策略进行优化, 这将是进一步研究的主要内容。

\section{参 考 文 献}

[1] CHAN C C, WONG Y S. The state of art of electric vehicles[J]. Journal of Asian Electric Vehicles, 2004(2): 
597-600.

[2] 赵治国, 何宁, 朱阳, 等. 四轮驱动混合动力轿车驱动 模式切换控制[J]. 机械工程学报, 2011, 47(4): 100-109. ZHAO Zhiguo, HE Ning, ZHU Yang, et al. Mode transition control for four wheel drive hybrid electric car[J]. Journal of Mechanical Engineering, 2011, 47(4): 100-109.

[3] AKIHIRO K, TETSUYA A, SHOICHI S. Driving forcecontrol of a parallel-series hybrid system[J]. JSAE Review, 1999, 20(3): 337-341.

[4] KAZUNARI M, YOSHIAKI I, YUKIO I. Design of the surge control method for the electric vehicle powertrain[R]. SAE, 2002-01-1935, 2002.

[5] HWANG H S, YANG D H. Torque control of engine clutch to improve the driving quality of hybrid electric vehicles[J]. International Journal of Automotive Technology, 2011, 12(5): 763-768.

[6] CANOVA M, GUEZENNEC Y, YURKOVICS H. On the control of engine start/stop dynamics in a hybrid electric vehicle[J]. Journal of Dynamic Systems, Measurement, and Control, 2009, 131: 061005-1-061005-12.

[7] 赵峰, 罗禹贡, 李克强, 等. 基于动态协调控制的 ISG 型混合动力电动汽车车引力控制方法 $[\mathrm{J}]$. 汽车工程, 2011(6): 463-467.

ZHAO Feng, LUO Yugong, LI Keqiang, et al. Traction control scheme for ISG hybrid electric vehicle based on dynamic coordinated control[J]. Automotive Engineering, 2011(6): 463-467.

[8] 童毅, 欧阳明高, 张俊智. 并联式混合动力汽车控制算
法的实时仿真研究 [J]. 机械工程学报, 2003, 39(10): 156-161.

TONG Yi, OUYANG Minggao, ZHANG Junzhi. Real-time simulation and research on control algorithm of parallel hybrid electric vehicle[J]. Chinese Journal of Mechanical Engineering, 2003, 39(10): 156-161.

[9] 严运兵. 并联混合动力电动汽车的动态控制研究 [D]. 武汉: 武汉理工大学, 2008.

YAN Yunbing. Study on the dynamic control in parallel hybrid electric vehicle[D]. Wuhan: Wuhan University of Technology, 2008.

[10] 戴一凡, 罗禹贡, 李克强, 等. 单电动机强混合动力电 动车辆的动态协调控制 [J]. 汽车工程, 2011(12): 1007-1012.

DAI Yifan, LUO Yugong, LI Keqiang, et al. Dynamic coordinated control for a full hybrid electric vehicle with single motor[J]. Automotive Engineering, 2011(12): 1007-1012.

[11] PISU P, RIZZONI G, CALO E. Control strategies for parallel hybrid electric vehicles[C]//IFAC Symp. Adv. Automot. Control, 2004: 508-513.

[12] LEE C C. Fuzzy logic in control system: Fuzzy logic controller. Part1[J]. IEEE Trans. on System Man and Cybernetics, 1990, 20(2): 404-418.

作者简介：倪成群(通信作者), 男, 1985 年出生, 博士研究生。主要研 究方向为新能源混合动力汽车电子控制。

E-mail: nichengqun@126.com

张幽四, 男, 1965 年出生, 博士, 教授, 博士研究生导师。主要研究方 向为车辆动力系统电子控制。

E-mail: youtong@bit.edu.cn 